\title{
Surface-Synthesized Graphene Nanoribbons for Room Temperature Switching Devices: Substrate Transfer and ex Situ Characterization
}

Gabriela Borin Barin, ${ }^{\dagger}$ Andrew Fairbrother, ${ }^{\dagger}$ Lukas Rotach, $^{\dagger}$ Maxime Bayle, ${ }^{\S}$ Matthieu Paillet, ${ }^{\S}$ Liangbo Liang, ${ }^{\|, \perp}$ Vincent Meunier, ${ }^{\perp}$ Roland Hauert, ${ }^{\ddagger}$ Tim Dumslaff, ${ }^{\#}$ Akimitsu Narita, \#৫

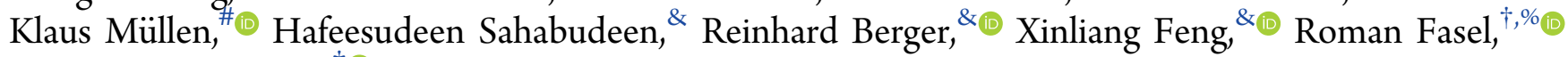
and Pascal Ruffieux ${ }^{*} \dagger$ (1)

$\dagger$ nanotech@surfaces Laboratory and $\doteqdot$ Joining Technologies and Corrosion Laboratory, Empa, Swiss Federal Laboratories for Materials Science and Technology, 8600 Dübendorf, Switzerland

${ }^{\S}$ Laboratoire Charles Coulomb (L2C), CNRS, Université de Montpellier, Montpellier, France

"Center for Nanophase Materials Sciences, Oak Ridge National Laboratory, Oak Ridge, Tennessee 37831, United States

${ }^{\perp}$ Department of Physics, Applied Physics, and Astronomy, Rensselaer Polytechnic Institute, Troy, New York 12180, United States

\#Max Planck Institute for Polymer Research, Ackermannweg 10, D-55128 Mainz, Germany

${ }^{\&}$ Chair of Molecular Functional Materials, Department of Chemistry and Food Chemistry, Technische Universität Dresden, Mommsenstrasse 4, Dresdan, Germany

${ }^{\%}$ Department of Chemistry and Biochemistry, University of Bern, Freiestrasse 3, CH-3012 Bern, Switzerland

\section{Supporting Information}

7-AGNR
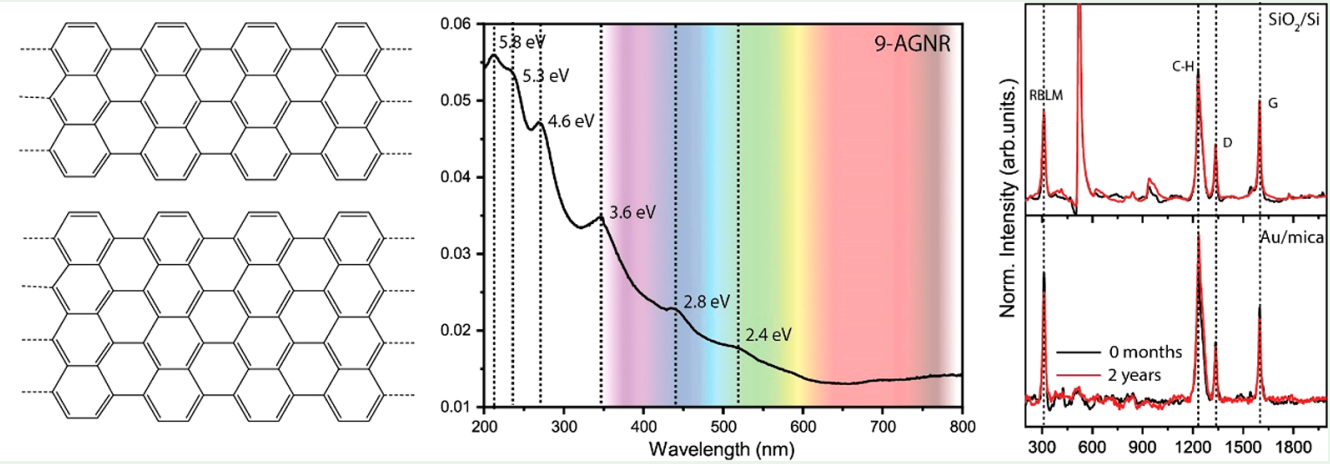

ABSTRACT: Recent progress in the on-surface synthesis of graphene nanoribbons (GNRs) has given access to atomically precise narrow GNRs with tunable electronic band gaps which makes them excellent candidates for room temperature switching devices such as field-effect transistors (FET). However, in spite of their exceptional properties, significant challenges remain for GNR processing and characterization. This contribution addresses some of the most important challenges, including GNR fabrication scalability, substrate transfer, long-term stability under ambient conditions, and ex situ characterization. We focus on 7- and 9-atom-wide armchair graphene nanoribbons (i.e., 7-AGNR and 9-AGNR) grown on $200 \mathrm{~nm} \mathrm{Au(111)/mica} \mathrm{substrates}$ using a high throughput system. Transfer of both 7- and 9-AGNRs from their Au growth substrate onto various target substrates for additional characterization is accomplished utilizing a polymer-free method that avoids residual contamination. This results in a homogeneous GNR film morphology with very few tears and wrinkles, as examined by atomic force microscopy. Raman spectroscopy indicates no significant degradation of GNR quality upon substrate transfer and reveals that GNRs have remarkable stability under ambient conditions over a 24 month period. The transferred GNRs are analyzed using multiwavelength Raman spectroscopy, which provides detailed insight into the wavelength dependence of the width-specific vibrational modes. Finally, we characterize the optical properties of 7- and 9-AGNRs via ultraviolet-visible (UV-vis) spectroscopy.

KEYWORDS: graphene nanoribbons, substrate transfer, multiwavelength Raman spectroscopy, scanning tunneling microscopy, atomic force microscopy, optical properties

\section{INTRODUCTION}

Atomically precise graphene nanoribbons have been extensively studied due to their remarkable electronic and optical
Received: January 24, 2019

Accepted: March 20, 2019

Published: March 20, 2019 


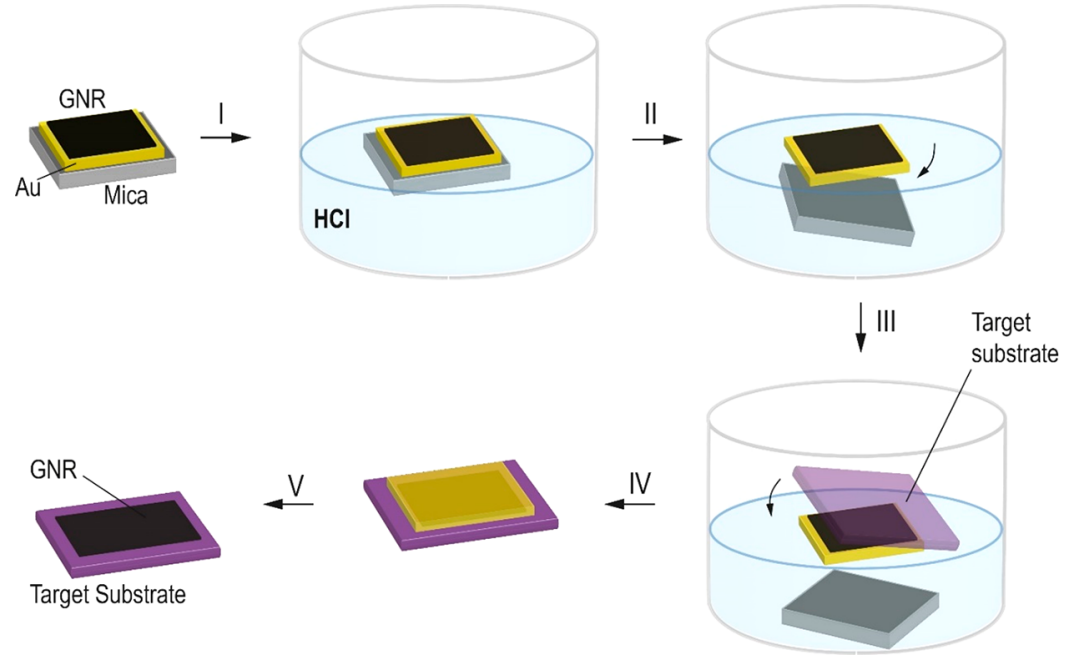

Figure 1. Scheme describing the basic steps of the polymer-free GNR transfer procedure. Step I: floating GNR/Au(111)/mica samples on aqueous $\mathrm{HCl}$ solution. Step II: cleavage of GNR/Au film from mica substrate ( $15 \mathrm{~min})$. Step III: target substrate is pushed onto the floating GNR/ $\mathrm{Au}(111)$ film. Step IV: increase adhesion between the Au film and the target substrate. Step V: etching of Au film resulting in GNRs on target substrate.

properties. $^{1-4}$ Because of lateral confinement, GNRs exhibit a sizable electronic band gap which can be tuned by their width and edge topology. ${ }^{1,5,6}$ To achieve such control over the band gap, atomic precision in the fabrication of the GNRs is indispensable. A bottom-up approach based on surface-assisted covalent coupling of molecular precursors ${ }^{1}$ allows for the synthesis of ultranarrow GNRs with defined edge topology and uniform width ${ }^{1,6,7}$ which results in an electronically homogeneous material across the entire growth substrate. The possibility of fabricating uniform, atomically precise GNRs with a tunable band gap makes this material promising for nanoelectronic and optoelectronic applications. ${ }^{3,4,8}$

Production is, however, just one aspect of a GNR technology, and to evaluate and exploit their properties, GNRs must be transferred onto semiconducting or insulating substrates. Different transfer techniques have been developed to transfer graphene and other 2D materials, which could potentially be adapted to graphene nanoribbons. ${ }^{9-12}$ The most widely used transfer procedure is the polymer assisted transfer (mainly using poly(methyl methacrylate) (PMMA)), ${ }^{13}$ in which graphene or GNRs samples are supported by a thin PMMA layer before the growth substrate is etched away. In general, the polymer-supported transfer is an easy-to-handle process, even though the complete removal of the polymer support remains a challenge. Solvent and thermal treatments have been used for this purpose, but such processes can induce thermal stress, which can damage the final transferred material. ${ }^{14}$ Because the purity and integrity of the GNR surface are of extreme importance for studying intrinsic properties and for GNR integration in nanoelectronic devices-such as field effect transistors ${ }^{4,8,15}$ - alternative methods need to be investigated.

Here we use a polymer-free process, previously reported by Cai et al. ${ }^{16}$ and Fairbrother et al., ${ }^{4}$ which allows the transfer of high quality, structurally intact GNRs. Using this transfer approach, we avoid any polymer residue resulting in a contamination-free transfer with fewer defects (wrinkles and tears) and preserved edge topology. Transferring GNRs to arbitrary substrates opens up the possibility of exploring GNR properties by characterization techniques that cannot be applied to metal-supported GNRs where intrinsic GNR properties are masked by the metallic substrate. Within this framework, it is of critical importance to develop reliable characterization protocols that allow monitoring GNR quality and stability upon transfer and device integration.

Here, we report an unprecedentedly detailed characterization of transferred GNRs including their morphological and spectroscopic properties using atomic force microscopy (AFM), Raman spectroscopy, and ultraviolet-visible spectroscopy (UV-vis). Using AFM, we investigate the overall morphology of transferred GNR films and evaluate our polymer-free transfer procedure. Raman spectroscopy results show that GNRs remain structurally intact upon transfer and have a remarkable stability under ambient conditions, as tracked over a 24 month period. Additionally, multiwavelength Raman measurements of GNRs reveal a unique nondispersive behavior in a large range of excitation energies. Ex situ characterization furthermore allows for unprecedented insights into the optical properties of GNRs. Using multilayer GNR films realized through repeated transfer sequences onto suitable substrates, we determine the fundamental optical transitions of 7- and 9-AGNRs via UV-vis spectroscopy.

\section{ON-SURFACE SYNTHESIS OF 7- AND 9-AGNRS}

Samples were produced in a stand-alone ultrahigh-vacuum (UHV) system dubbed a "GNR reactor" that has been specifically designed to allow for a fully automatized, reproducible, and high-throughput fabrication of GNR samples (see Figure S1 for GNR reactor details). The fast and reproducible fabrication of $\mathrm{GNR} / \mathrm{Au}(111) /$ mica samples in the GNR reactor is necessary to produce the large number of samples needed for efficient optimization of transfer procedures and ex situ characterization of well-defined GNR samples. As a growth substrate we used commercially available $\mathrm{Au}(111)$ thin films supported on mica $\left(4 \times 4 \mathrm{~mm}^{2}, 200 \mathrm{~nm}\right.$ $\mathrm{Au}$; PHASIS, Geneva, Switzerland).

9-AGNRs were synthesized from $3^{\prime}, 6^{\prime}$-di-iodine-1, $1^{\prime}: 2^{\prime}, 1^{\prime \prime}$ terphenyl (DITP) as the precursor monomer ${ }^{5}$ and 7-AGNR from 10,10' -dibromo-9, $9^{\prime}$-bianthryl (DBBA). ${ }^{1}$ First, the $\mathrm{Au}(111) /$ mica substrate is cleaned in ultrahigh vacuum by 

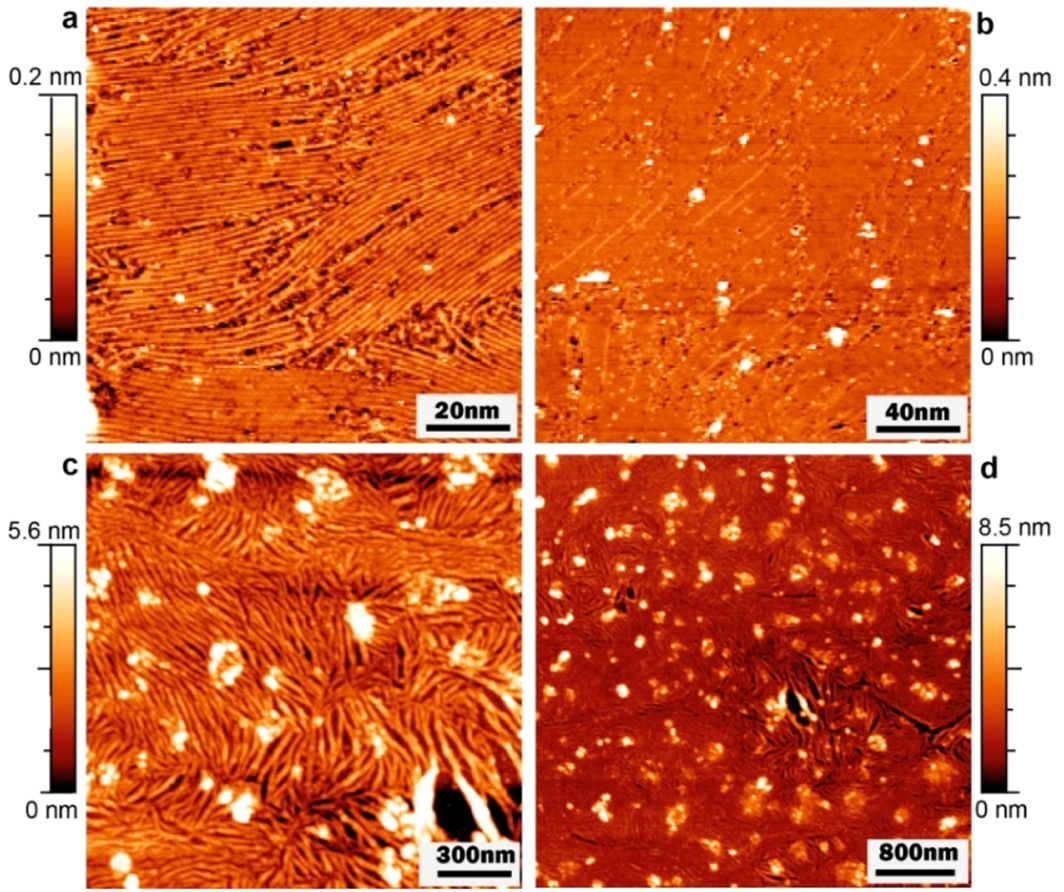

Figure 2. (a, b) STM topography images of 9-AGNRs on Au/mica (RT, $1.5 \mathrm{~V}, 0.02 \mathrm{nA}$ ) and (c, d) AFM topography images of 9-AGNRs transferred onto an $\mathrm{Al}_{2} \mathrm{O}_{3}$ substrate.

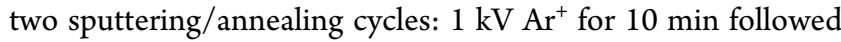
by annealing at $470{ }^{\circ} \mathrm{C}$ for $10 \mathrm{~min}$. Next, the monomer is sublimed onto the $\mathrm{Au}(111)$ surface from a quartz crucible heated to $70{ }^{\circ} \mathrm{C}$ (DITP) or $200{ }^{\circ} \mathrm{C}$ (DBBA) with the substrate held at room temperature. After $4 \mathrm{~min}$ of deposition (resulting in $\sim 1$ monolayer coverage $)$, the substrate is heated $\left(0.5^{\circ} \mathrm{C} / \mathrm{s}\right)$ up to $200{ }^{\circ} \mathrm{C}$ with a $10 \mathrm{~min}$ holding time to activate the polymerization reaction, followed by annealing at $400{ }^{\circ} \mathrm{C}(0.5$ ${ }^{\circ} \mathrm{C} / \mathrm{s}$ with a $10 \mathrm{~min}$ holding time) to form the GNRs via cyclodehydrogenation of the polyphenylene precursors.

\section{TRANSFER PROCEDURE}

To explore the GNRs' properties under various conditions, we have transferred the GNRs from the $\mathrm{Au}(111) /$ mica growth substrate onto different target substrates. In this work we use a polymer-free method, ${ }^{4,16}$ as illustrated in Figure 1. Compared to polymer-assisted transfer procedures used in previous works, our method leads to a clean substrate transfer with low density of tears and wrinkles in the GNR film. ${ }^{8,14,17}$ These aspects are of critical importance for the integration of GNRs into FETs and for achieving high device performance. First, the GNR/ $\mathrm{Au}(111) /$ mica samples are floated on aqueous $\mathrm{HCl}$ solution (38\%, room temperature, $3.5 \mathrm{~mL}$ ) with the GNR/Au film facing up (step I in Figure 1). After $\sim 15$ min the mica cleaves off from the Au film due to the etching of the interface by $\mathrm{HCl}$ (step II in Figure 1). After the mica is detached, $3 \mathrm{~mL}$ of acid is removed and $5 \mathrm{~mL}$ of ultrapure water is added. This step is repeated five times to substantially reduce the $\mathrm{HCl}$ concentration.

Prior to transfer of the GNRs, any impurities need to be removed from the target substrate. This is achieved through sonication in acetone and ethanol for $10 \mathrm{~min}$ followed by rinsing with ultrapure water. In the next step, the target substrate is pushed onto the floating GNR/Au(111) film (step III in Figure 1), which results in the adherence of the GNR/Au film on the target substrate. At this stage, the Au film is usually not completely flat on the substrate. To increase adhesion between the Au film and the target substrate, we apply a drop of ethanol to the top of the Au film (dried at ambient conditions for $5 \mathrm{~min}$ ) followed by a hot plate annealing step at $100{ }^{\circ} \mathrm{C}$ for $10 \mathrm{~min}$ (step IV in Figure 1; see also Figure S2 for step IV details). Finally, 1-2 drops of potassium iodide-based gold etchant (potassium iodine, no dilution) is used to etch away the gold film ( $\sim 5 \mathrm{~min})$. The resulting GNR/substrate sample is cleaned by soaking it in ultrapure water for $5 \mathrm{~min}$ followed by acetone/ethanol rinsing and finally dried with nitrogen (step V in Figure 1). This polymer-free process results in a large area transfer of GNRs onto arbitrary target substrates while fully suppressing the risk of having polymer residues on the transferred GNRs.

X-ray photoelectron spectroscopy (XPS) measurements were performed to ensure the effectiveness of the cleaning process (with organic solvents and ultrapure water after the $\mathrm{Au}$ etching step). As shown in Figure S3, 9-AGNRs transferred to $\mathrm{CaF}_{2}$ and $\mathrm{Al}_{2} \mathrm{O}_{3}$ have very small iodine contributions corresponding to $\mathrm{ca}$. $0.3 \%$ and $0.2 \%$ of a monolayer, respectively. GNRs transferred to a $\mathrm{SiO}_{2} / \mathrm{Si}$ substrate showed no clear iodine signal, an indication of iodine to be below the detection limit of ca. $0.1 \%$ of a monolayer.

\section{GRAPHENE NANORIBBON CHARACTERIZATION}

Morphology of As-Grown and Transferred GNR Films. After growth, 9-AGNR samples were removed from the GNR reactor and inserted in another UHV system for STM characterization. To desorb contaminants accumulated during ambient exposure, the samples were annealed at $200{ }^{\circ} \mathrm{C}$ for $20 \mathrm{~min}$. High-resolution STM images taken at room temperature reveal 9-AGNRs with an average length of $\sim 45$ $\mathrm{nm}$ (Figure 2a). To make a direct comparison of the topography of the GNR film before and after substrate transfer, a large-scale STM image from a 9-AGNR/Au/mica sample is displayed in Figure $2 \mathrm{~b}$. It reveals the growth 
uniformity as well as the quasi-continuous film-like morphology that is related to the high coverage preparation.

After transfer to $\mathrm{Al}_{2} \mathrm{O}_{3}$ substrates, atomic force microscopy shows uniform and smooth 9-AGNR-films (roughness $\sim 1-2$ $\mathrm{nm}$; see Figure 2c,d and Figure S4 for the roughness profile). High-resolution AFM (at ambient conditions) reveals a high coverage of GNRs bundles (2-4 ribbons) across the whole scan area (Figure 2c). As observed in Figure $2 \mathrm{~d}$, the transferred GNR film contains relatively few tears, wrinkles, and folds in comparison with polymer-assisted transfer., ${ }^{9,14,18}$ The observed wrinkles and folds in the GNR film are related to folding of the $\mathrm{Au}$ film during the transfer process (see Figure S2 for transfer details). This direct comparison of STM and AFM images is the first indication that the polymer-free transfer procedure allows for the uniform transfer of GNRs from $\mathrm{Au}(111) / \mathrm{mica}$ onto e.g. $\mathrm{Al}_{2} \mathrm{O}_{3}$, which is crucial before exploring other nonvacuum characterization methods and the development of further processing steps for device integration. ${ }^{3}$

Post-Transfer Stability. Transferring the nanoribbons to $\mathrm{SiO}_{2} / \mathrm{Si}$ or other insulating substrates opens up the possibility of investigating the intrinsic properties of unperturbed GNRs. Among the substrates investigated in this work are $\mathrm{SiO}_{2} / \mathrm{Si}$ substrates that are technologically relevant for device fabrication and $\mathrm{Al}_{2} \mathrm{O}_{3}$ and $\mathrm{CaF}_{2}$ that are transparent and hence ideally suited to assess the optical properties of GNRs and transmission electron microscopy (TEM) grids which could potentially be used for atomic scale characterization of transferred GNRs. Raman spectroscopy is a straightforward way to demonstrate the integrity and stability of GNRs after the transfer procedure. The first-order Raman spectra of graphene and other $\mathrm{sp}^{2}$-bonded carbon-based materials present a main band at $\sim 1590 \mathrm{~cm}^{-1}$, the so-called $\mathrm{G}$ mode, ${ }^{19}$ which originates from in-plane vibrations and dominates the optical region of graphene-based materials. ${ }^{20-22}$ When defects are present, the Raman spectrum of graphene also shows a band at $\sim 1340 \mathrm{~cm}^{-1}$, known as the $\mathrm{D}$ mode, which is activated by an intervalley double-resonance process. Unlike graphene, for which the $\mathrm{D}$ band is excited by the presence of defects, ${ }^{23,24}$ GNRs have a D mode originating from a first-order process. ${ }^{19,25,26}$ Because of the edges, the $\mathrm{D}$ mode is an intrinsic excitation for GNRs and is expected to be nondispersive as the G mode. ${ }^{27}$ The $\mathrm{D}$ mode of GNRs is characterized by a sharp peak (FWHM $\sim 14 \mathrm{~cm}^{-1}$ ) around $\sim 1335-1340 \mathrm{~cm}^{-1}$. In addition to a well-structured D mode, GNR spectra also show modes at $\sim 1200-1300 \mathrm{~cm}^{-1}$ assigned to the $\mathrm{C}-\mathrm{H}$ bending modes localized at the edges. ${ }^{28}$ The lowenergy frequency range also brings valuable information for $\mathrm{sp}^{2}$ carbon with finite width. The radial-breathing mode (RBM) has been widely explored in carbon nanotubes to identify the tube's diameter. ${ }^{29}$ A similar mode called radial breathing-like mode (RBLM) was predicted to be related to edge structure and width for graphene nanoribbons. ${ }^{1,25,30}$ Sanders et al. ${ }^{31}$ indicated via tight-binding calculations that the RBLM of GNRs is coherently excited for photon energies near the GNR's lowest optical transitions. ${ }^{5,31}$ For the 9-AGNR, Prezzi et al. ${ }^{32}$ calculated this value to be $1.0 \mathrm{eV}$. For our Raman study, we used a $785 \mathrm{~nm}$ laser $(1.58 \mathrm{eV})$, which is sufficiently close to the optical band gap of 9-AGNRs to yield excitation of the RBLM. ${ }^{5}$ In agreement with the prediction by Vandescuren et al., ${ }^{30}$ we observe the RBLM of the 9 -AGNR at $311 \mathrm{~cm}^{-15}$ on the $\mathrm{Au}$ substrate (Figure 3a). After substrate transfer the RBLM does not show significant changes, indicating that GNRs remain structurally intact upon transfer (Figure 3a).
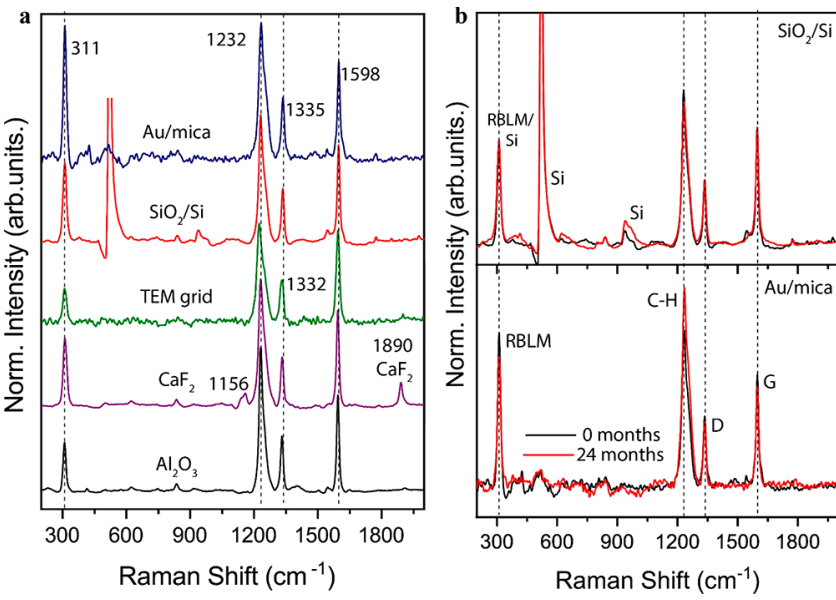

Figure 3. Raman analysis of 9-AGNRs on various substrates $(\lambda=785$ $\mathrm{nm}$ ). (a) 9-AGNRs on the Au/mica growth substrate and after transfer to $\mathrm{SiO}_{2} / \mathrm{Si}$, transmission electron microscopy (TEM) grid, $\mathrm{CaF}_{2}$ and $\mathrm{Al}_{2} \mathrm{O}_{3}$ substrates. (b) 9-AGNRs on the $\mathrm{Au} /$ mica growth substrate (bottom) and after transfer to a $\mathrm{SiO}_{2} / \mathrm{Si}$ wafer (top), taken immediately after growth/processing ("0 months", black curves) and after storage under ambient conditions for 24 months (red curves). Average of three spectra normalized to the $G$ mode intensity.

Moreover, no significant changes are observed for the main peaks of the first-order Raman spectra: $\mathrm{C}-\mathrm{H}$ mode (1232 $\left.\mathrm{cm}^{-1}\right)$, D mode $\left(1335 \mathrm{~cm}^{-1}\right)$ and $\mathrm{G}$ mode $\left(1598 \mathrm{~cm}^{-1}\right)$, showing that the GNR's atomically precise edges and the $\mathrm{sp}^{2}$ network are preserved upon substrate transfer.

In addition to demonstrating the robustness of GNRs during transfer, it is important to probe their stability over time under ambient conditions. Achieving long-term stability of GNRs would be a significant step toward their application as an active material in devices. Samples of 9-AGNRs on $\mathrm{Au}(111) / \mathrm{mica}$ and transferred to $\mathrm{SiO}_{2} / \mathrm{Si}$ substrates were stored under ambient conditions, and Raman spectra were recorded over a 24 month period. Figure $3 \mathrm{~b}$ shows Raman profiles acquired immediately after growth and transfer ( 0 months, in black) and after 24 months of storage (in red), normalized to the $\mathrm{G}$ mode intensity. For both samples ( 0 and 24 months) three different single spectra were acquired at different locations of the sample. The individual spectra exhibited the same Raman features with similar overall and relative intensities and were thus averaged to yield the spectra shown in Figure $3 \mathrm{~b}$. Compared with the spectra recorded immediately after synthesis on $\mathrm{Au}(111) / \mathrm{mica}$ and after transfer to $\mathrm{SiO}_{2} / \mathrm{Si}$, over time a small change in peak width is observed. For both the as-grown 9-AGNRs on $\mathrm{Au}(111) / \mathrm{mica}$ and the 9-AGNRs transferred to $\mathrm{SiO}_{2}$, the full width at half-maximum (FWHM) of the $\mathrm{D}$ mode is $11 \mathrm{~cm}^{-1}$ and increases slightly to $14 \mathrm{~cm}^{-1}$ after two years. Similarly, for the $G$ mode we see an increase of the FWHM from 13 to $16 \mathrm{~cm}^{-1}$ which could be associated with adsorbates (atmospheric contamination). ${ }^{4}$ The RBLM mode on $\mathrm{Au}$ did not show any significant difference over the 24 month period. For the transferred sample, a detailed analysis of the RBLM mode is difficult since it falls into the region of the Si mode $\left(302 \mathrm{~cm}^{-1}\right)$.

For 9-AGNRs, C-H, D, and G peak shifts remain below the experimental accuracy $\left(3 \mathrm{~cm}^{-1}\right)$ of the Raman experiments. On the basis of these findings, we can affirm that the 9-AGNRs have remarkable stability with no significant structural modifications even after prolonged exposure to air. Such 

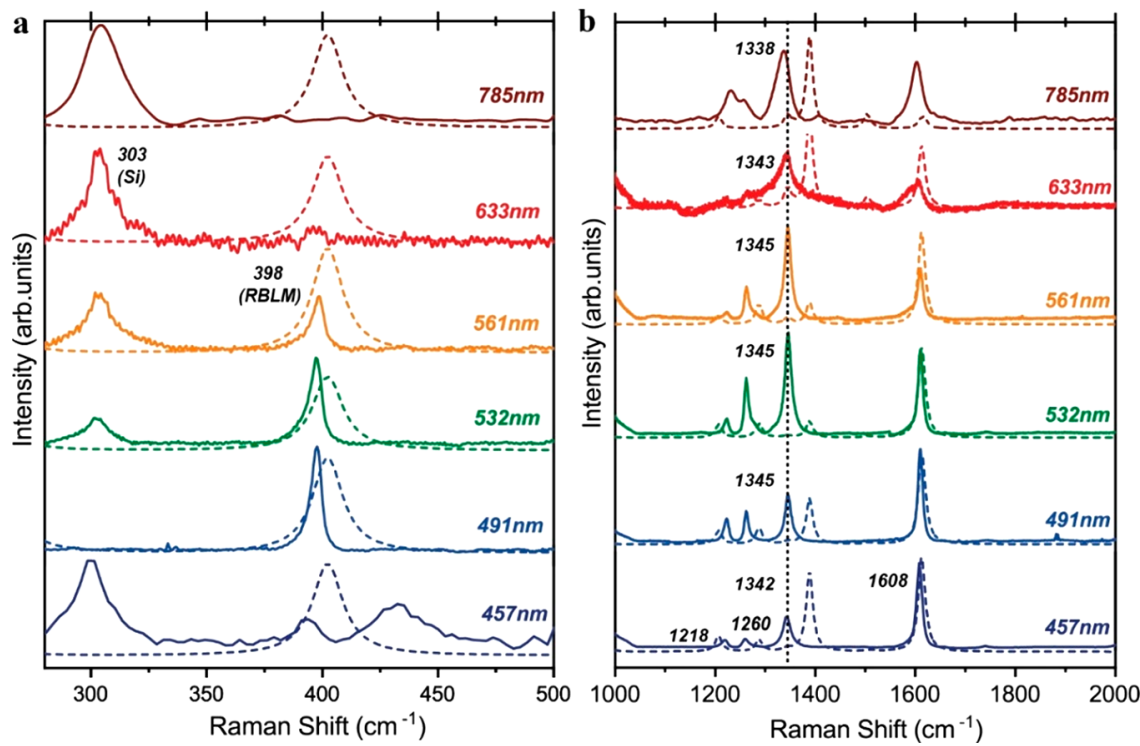

Figure 4. Multiwavelength Raman study of 7-AGNRs (at 0.5 monolayer coverage) transferred to $\mathrm{SiO}_{2} / \mathrm{Si}$ : (a) low-frequency region; (b) high frequency region (solid line: experimental data; dashed line: simulated spectra). Spectra are not normalized.
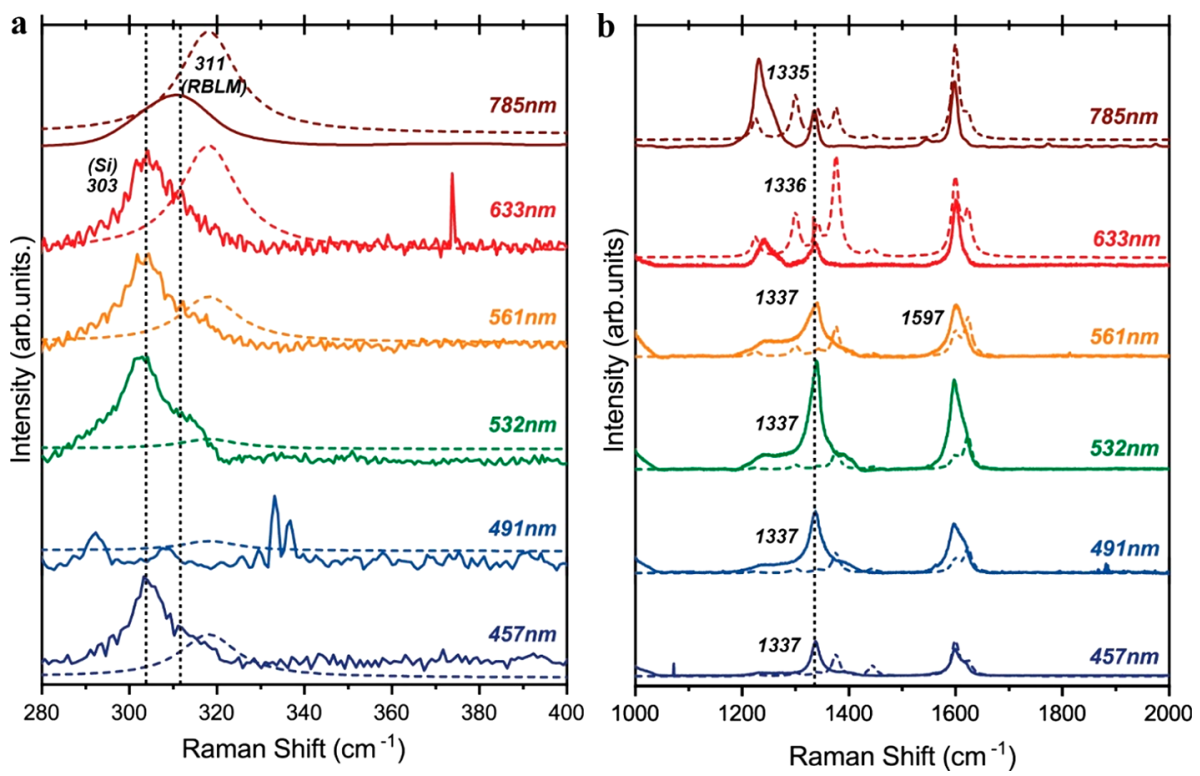

Figure 5. Multiwavelength Raman study of 9-AGNRs (at 0.5 monolayer coverage) transferred to $\mathrm{SiO}_{2} / \mathrm{Si}$ ): (a) low frequency region; (b) high frequency region (solid line: experimental data; dashed line: simulated spectra). Spectra are not normalized.

long-term stability is a key requirement for the further exploitation of GNRs in applications, and 9-AGNRs have proven to be the most stable GNR system reported so far.

Multiwavelength Raman Spectroscopy. The unique nature of graphene nanoribbons - with their quasi-infinite length, finite width, and atomically precise edges-offers a very interesting testbed to investigate well-defined vibrational properties. GNRs with armchair edges and semiconducting behavior do not have Kohn anomalies or linear electronic dispersion. ${ }^{2,20,32}$ In the case of cove-GNRs, for example, the $K$ point folds onto $\Gamma^{33}$ resulting in a first-order process for activating the $\mathrm{D}$ mode, and therefore a nondispersive behavior is expected. The latter differentiates the Raman spectra of GNRs from graphene in which the D mode is activated by an intervalley double-resonance process in the presence of defects and shows a strong dispersion in function of the excitation energy. $^{24,25,34}$

To investigate the (non)dispersive behavior of the Raman features of AGNRs, both 7- and 9-AGNRs transferred to $\mathrm{SiO}_{2} /$ $\mathrm{Si}$ substrates were measured with excitation energies ranging from 785 to $457 \mathrm{~nm}$ (1.57 to $2.7 \mathrm{eV}$, Figures 4 and 5, respectively). The $\mathrm{D}$ mode of both 7 - and 9-AGNRs is nondispersive (maximum D mode shift of $5 \mathrm{~cm}^{-1}$ ), indicating the absence of disorder-induced scattering for these substratetransferred ribbons (see Figures S5 and S6 for deconvoluted spectra and Figure S7 for dispersion plots). This further confirms that our polymer-free transfer does not induce defects or edge functionalization. In addition, 7-AGNRs reveal nondispersive behavior for the RBLM peak, observed at 396 $\mathrm{cm}^{-1,1,4}$ and for both $\mathrm{C}-\mathrm{H}$ related modes at $\sim 1220$ and $\sim 1262 \mathrm{~cm}^{-1}$, showing that both width and edge structure are 
a

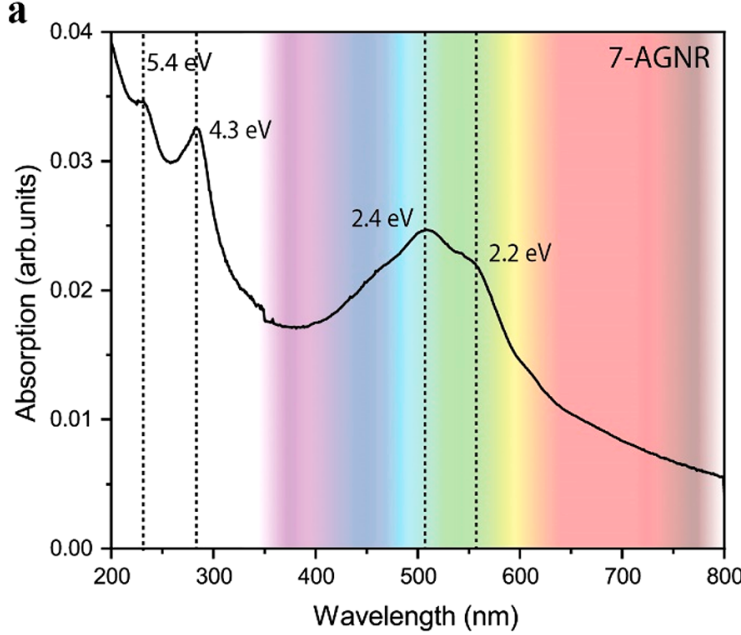

b

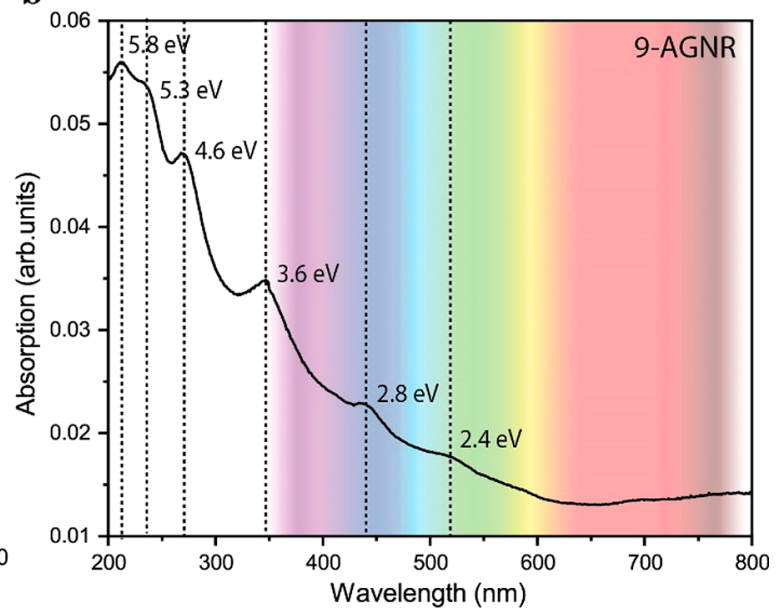

Figure 6. (a) UV-vis spectrum of four layers of 7-AGNR transferred to $\mathrm{Al}_{2} \mathrm{O}_{3}$. (b) UV-vis spectrum of four layers of 9-AGNR transferred to $\mathrm{Al}_{2} \mathrm{O}_{3}$.

preserved upon transfer. It was not possible to evaluate the dispersion of the RBLM for the 9-AGNR from our experimental data since this mode is only observed with the $785 \mathrm{~nm}$ excitation wavelength, which falls nearest to the lowest optical transition of the 9-AGNR $(\sim 1 \mathrm{eV}) .{ }^{31,32}$

Raman spectra measured for both 7- and 9-AGNR were compared to first-principles Raman simulations that take the dependence on the excitation wavelength into account. For both 7- and 9-AGNRs, we observe an overall agreement between experimental and simulated spectra (Figures 4 and 5). Experimental and simulated Raman spectra show a strong dependence on excitation wavelength with respect to both overall and relative intensities of the different modes. This behavior originates from the resonant Raman process in which the excitation wavelength overlaps with (or is very close to) an optical transition. Therefore, increased scattering intensities are expected, making it possible to experimentally access the radial breathing-like mode and edge-related modes of 7-AGNRs and 9-AGNRs even at submonolayer coverage.

Additionally, for the 9-AGNR we observe a splitting of the G band with a strong dependence of the relative intensities on the excitation energy for both experimental and simulated spectra. Theoretically, the two G-band components are due to a splitting of the doubly degenerate $G$ mode of extended graphene induced by the finite size and in-plane anisotropy in 9-AGNRs. ${ }^{5}$ According to the normal-mode analysis (Figure S8), this splitting gives rise to two Raman-active modes: the G1 mode at $1599 \mathrm{~cm}^{-1}$ with $A_{g}$ symmetry and a second G2 mode at $1623 \mathrm{~cm}^{-1}$ with $B_{1 g}$ symmetry. For the 7-AGNR, calculations also indicate two $\mathrm{G}$ modes with $\mathrm{G} 1$ at $1612 \mathrm{~cm}^{-1}$ and G2 at $1619 \mathrm{~cm}^{-1}$ (Figure S8). The simulated separation of G1 and G2 modes for the 7-AGNR is about $7 \mathrm{~cm}^{-1}$ and can be observed as a broadening of the $\mathrm{G}$ peak measured from 561 to $785 \mathrm{~nm}$ (Figure 4 and Figure S5).

Finally, we note that there are some discrepancies in the overall intensity of the peaks between the experimental and simulated spectra. Raman intensities are sensitive to many factors, such as excitonic and substrate effects. In addition, the interface between GNRs and $\mathrm{SiO}_{2} / \mathrm{Si}$ substrate causes multiple reflections and interference effects. ${ }^{35-37}$ These effects are difficult to model and not included in the present simulations, resulting in differences in the relative peak intensities between experimental and simulated spectra. Nonetheless, the simulated spectra provide insights into the intrinsic Raman properties of isolated AGNRs free from external effects.

UV-Vis Spectroscopy. Owing to the pronounced width dependence of the electronic structure of GNRs, the optical absorption can also be tuned over a wide range. For small band gap GNRs, optical absorption can even reach the near-infrared (NIR) region, opening up possibilities for GNRs in technologies such as photovoltaics. ${ }^{33}$ Here, 7- and 9-AGNRs have been transferred to (transparent) $\mathrm{Al}_{2} \mathrm{O}_{3}$ substrates to investigate their absorption spectrum in the $\mathrm{UV}-$ vis range $(200-800 \mathrm{~nm})$ in transmission geometry.

UV-vis absorption spectra of 7-AGNRs and 9-AGNRs are shown in Figure 6. To increase the intensity of absorption features, an $\mathrm{Al}_{2} \mathrm{O}_{3}$ sample with four layers of GNRs was measured. The four-layer coverage was achieved by a sequential repetition of the transfer process described in Figure 1.

For the 7-AGNR we observed strong absorption bands with local maxima at 2.2, 2.4, 4.3, and $5.4 \mathrm{eV}(560,510,285$, and $230 \mathrm{~nm}$, respectively). Denk et al. ${ }^{2}$ computed the optical response of isolated 7-AGNRs which revealed three main absorption features located at $1.9,2.3$, and $4.1 \mathrm{eV}$. These are in good agreement with the experimental values obtained in this work (Figure 6). The two low-energy excitonic contributions at 2.2 and $2.4 \mathrm{eV}$ are assigned to optical transitions between the last valence band and first conduction band $\left(E_{11}\right)$ and between the next to last valence band and second conduction band $\left(E_{22}\right)$, respectively. ${ }^{2}$ In addition, the absorption bands are in very close agreement to the ones determined for metaladsorbed 7-AGNRs $(2.1,2.3$, and $4.2 \mathrm{eV}){ }^{2}$ indicating negligible substrate contributions to the optical response of AGNRs.

For the 9-AGNR we observe strong absorption bands between 200 and $600 \mathrm{~nm}$, with local maxima at 212, 233, 270, 347,440 , and $520 \mathrm{~nm}$ (i.e., 5.8, 5.3, 4.6, 3.6, 2.8, and $2.4 \mathrm{eV}$, respectively). It is clear that the first optical excitation predicted to be around $1.0 \mathrm{eV}^{32}$ is not accessible for this detection range and that the observed absorption features are thus related to higher order optical transitions. However, the absorption features observed at 2.4, 2.8, and $3.6 \mathrm{eV}$ agree well 
with the predicted higher-order transitions at 2.4, 2.9, and 3.3 $\mathrm{eV}^{32}$

\section{CONCLUSIONS}

In this work, we report substrate transfer and post-transfer characterization of 7- and 9-AGNRs. AFM imaging reveals that the applied polymer-free transfer procedure yields clean and uniform large area GNR films. Raman characterization before and after GNR substrate transfer indicates that width and edge topology are preserved upon transfer onto different target substrates. In addition, transferred GNRs show remarkable stability over time under ambient conditions, validating their potential for integration in electronic devices. Multiwavelength Raman measurements further confirm the quality of transferred 7- and 9-AGNRs and experimentally validate the theoretically predicted nondispersive behavior of all Raman modes. Finally, UV-vis spectroscopy is used to determine the absorption spectrum. For 9-AGNRs, strong absorption bands across a wide energy range ( 6.2 to $2.3 \mathrm{eV}$ ) are found, while 7-AGNRs present high-intensity absorption features at 2.2, 2.4, and 4.3 $\mathrm{eV}$, revealing the marked width dependence of GNR's optical properties. Altogether, detailed ex situ characterization allowed us to obtain unprecedented insights into transfer uniformity, stability, and quality of GNRs, which are key factors toward the technological application of graphene nanoribbons.

\section{MATERIALS AND METHODS}

Au/Mica Substrates. Au/mica substrates were purchased from Phasis (Geneva, Switzerland). They consist of Au (111) films, with $200 \mathrm{~nm}$ thickness and $4 \times 4 \mathrm{~mm}^{2}$ dimensions, epitaxially grown on muscovite mica sheets by magnetron sputtering. The freshly cleaved mica used as substrate for the Au films has a thickness of around $0.080-0.130 \mathrm{~mm}$. Mica is a silicate material with nearly perfect basal cleavage and is an excellent substrate for growing high-quality quasisingle crystalline $\mathrm{Au}(111)$ films.

$\mathrm{Al}_{2} \mathrm{O}_{3}$ and $\mathrm{CaF}_{2}$ Substrates. $\mathrm{Al}_{2} \mathrm{O}_{3}$ and $\mathrm{CaF}_{2}$ were purchased from Crystal $\mathrm{GmbH}$, Germany. Both substrates were polished on both sides and have dimensions of $7 \times 7 \times 0.5 \mathrm{~mm}^{3}$. Both substrates were scratch-free under magnification of $50 \times$ (surface quality given by the provider).

$\mathrm{SiO}_{2} / \mathrm{Si}$ Substrates. $\mathrm{SiO}_{2} / \mathrm{Si}$ substrates were purchased from Nova Electronics. They consist of $285 \mathrm{~nm}$ dry chlorinated thermal oxide on $525 \mu \mathrm{m}\langle 100\rangle$ directional silicon. The 4 in. wafer was cut in smaller substrates with $7 \times 7 \mathrm{~mm}^{2}$ dimensions.

TEM Grids. TEM grids were purchased from Ted Pella, Inc. They consist of monolayer graphene TEM support films suspended on a lacey carbon film on a 300 mesh grid.

Au Etchant. Potassium iodide-based gold etchant was purchased from Sigma-Aldrich CAS No. 7681-11-0 and used with no further dilution.

X-ray Photoelectron Spectroscopy (XPS). XPS was performed in a Physical Electronics Instruments Quantum 2000 spectrometer using monochromatic $\mathrm{Al} \mathrm{K} \alpha$ radiation generated from an electron beam operated at 15 and $32.3 \mathrm{kV}$. Spectra were collected under ultrahigh-vacuum conditions (residual pressure $=5 \times 10^{-8} \mathrm{~Pa}$ ) at an analyzer pass energy of $46.95 \mathrm{eV}$ to yield a total analyzer energy resolution of $0.95 \mathrm{eV}$ (for $\mathrm{Ag} 3 \mathrm{~d}$ electrons). For compensation of possible sample charging, an electron and Ar-ion neutralizer were simultaneously used. The spectrometer energy scale was calibrated for the $\mathrm{Au} 4 \mathrm{f}_{7 / 2}$ signal to be at $84.0 \pm 0.1 \mathrm{eV}$

Scanning Tunneling Microscopy (STM). Topographic scanning tunneling microscopy images of as-grown 9-AGNRs on $\mathrm{Au} / \mathrm{mica}$ samples were taken with a Scienta Omicron VT-STM operated at room temperature. Constant-current STM images were recorded with $1.5 \mathrm{~V}$ sample bias and $0.02 \mathrm{nA}$ set point current.
Atomic Force Microscopy (AFM). The topography of substrate transferred graphene nanoribbon samples was characterized using tapping mode AFM (Bioscope, Bruker) with silicon probes from OPUS (model 160AC-SG Ultrasharp cantilever) with tip radius $<1$ $\mathrm{nm}$ (force constant $\sim 26 \mathrm{~N} \mathrm{~m}^{-1}$, resonance frequency in the range of $300 \mathrm{kHz}$ ). Height diagrams were recorded with scan sizes of $500 \mathrm{~nm}$, $1.5 \mu \mathrm{m}$, and $5 \mu \mathrm{m}$ and scan speeds of $1 \mathrm{~Hz}(512 \times 512$ points $)$. The $\mathrm{WSxM}^{38}$ software was used for AFM analysis.

Raman Spectroscopy. Raman spectroscopy with laser wavelength of $785 \mathrm{~nm}$ was performed with a Bruker SENTERRA Raman microscope, operated at $10 \mathrm{~mW}$ laser power. Spectra were acquired by three sweeps with $10 \mathrm{~s}$ of integration time and a $50 \times$ objective lens. Multiwavelength Raman spectroscopy $(457-633 \mathrm{~nm})$ was performed with an Acton SP2500 spectrometer fitted with a Pylon CCD detector and a grating of 1800 grooves $/ \mathrm{mm}$. The samples were excited through a $100 \times$ objective (Numerical Aperture 0.9) with power ranging from 0.5 to $2 \mathrm{~mW}$ and 1 sweep with $10 \mathrm{~s}$ integration time. The lasers used are at $532 \mathrm{~nm}$ (Millennia Prime, Newport), at 457, 491, and $561 \mathrm{~nm}$ (Cobolt DPSS), and at $633 \mathrm{~nm}(\mathrm{He}-\mathrm{Ne}$, Newport).

Computational Procedures. Plane-wave DFT calculations were performed using the VASP package ${ }^{39}$ with local density approximation (LDA) exchange-correlation functionals and projector augmented-wave (PAW) pseudopotentials. The energy cutoff was set at $500 \mathrm{eV}$. For one-dimensional 9-AGNR and 7-AGNR, the lattice constant in the periodic direction (i.e., $x$ direction) is $a=4.256 \AA$. In the nonperiodic directions ( $y$ and $z$ directions), a vacuum region of about $18 \AA$ was used to avoid spurious interactions with periodic replicas. For the unit cell, $24 \times 1 \times 1 k$-point sampling was used, and all atoms were relaxed until the residual forces were below $0.001 \mathrm{eV} /$ A. Phonon calculations were then performed using the finite difference scheme implemented in the PHONON software. ${ }^{40}$ The Hellmann-Feynman forces in the $3 \times 1 \times 1$ supercell were computed using VASP for both positive and negative atomic displacements $(\delta=$ $0.03 \AA$ ) and then used in PHONON to construct the dynamic matrix, whose diagonalization provides the phonon frequencies and eigenvectors (i.e., vibrations). Raman intensity calculations were then performed within the Placzek approximation ${ }^{5,41,42}$ (more details in the Supporting Information).

UV-Vis Spectroscopy. UV-vis spectra were measured on a Cary $5000 \mathrm{UV}$-vis instrument with a sample holder with $1 \mathrm{~mm}$ mask at room temperature. Absorption/transmission spectra from 200 to 800 $\mathrm{nm}$ were recorded at a scan rate of $30 \mathrm{~nm} \mathrm{~min} \mathrm{mi}^{-1}$ (data integral: $1 \mathrm{~nm}$ ). The spectral bandwidth was set to $5 \mathrm{~nm}$. The substrate reference spectrum was ran separately and later subtracted from the GNRs absorption spectra.

\section{ASSOCIATED CONTENT}

\section{S Supporting Information}

The Supporting Information is available free of charge on the ACS Publications website at DOI: 10.1021/acsanm.9b00151.

Description of the GNR reactor, additional information about the substrate transfer method, XPS and AFM data of transferred GNRs and supplementary Raman analysis including the fitting of deconvoluted spectra and dispersion plots of the multiwavelength Raman measurements and normal-mode analysis (PDF)

\section{AUTHOR INFORMATION}

\section{Corresponding Author}

*E-mail: pascal.ruffieux@empa.ch. ORCID

Gabriela Borin Barin: 0000-0002-1069-6182

Akimitsu Narita: 0000-0002-3625-522X

Klaus Müllen: 0000-0001-6630-8786

Reinhard Berger: 0000-0002-8959-7821 
Xinliang Feng: 0000-0003-3885-2703

Roman Fasel: 0000-0002-1553-6487

Pascal Ruffieux: 0000-0001-5729-5354

\section{Present Addresses}

A.F.: Engineering Laboratory, National Institute of Standards and Technology (NIST), Gaithersburg, MD 20899.

M.B.: Institut des Matériaux Jean Rouxel (IMN), Université de Nantes, CNRS, 2 rue de la Houssinière, BP 32229, 44322 Nantes cedex 3, France.

\section{Notes}

The authors declare no competing financial interest.

\section{ACKNOWLEDGMENTS}

This work was supported by the Swiss National Science Foundation under Grant No. 20PC21_155644, the European Union's Horizon 2020 research and innovation programme under Grant Agreement No. 785219 (Graphene Flagship Core 2 ), and the Office of Naval Research BRC Program under Grant No. N00014-12-1-1009. Part of this work (Raman scattering modeling) used resources at the Center for Nanophase Materials Sciences, which is a DOE Office of Science User Facility operated by the Oak Ridge National Laboratory. L.L. was supported by a Eugene P. Wigner Fellowship and by the Center for Nanophase Materials Sciences. Part of the computations was performed using resources of the Center for Computational Innovation at Rensselaer Polytechnic Institute. H.S., R.B., and X.F. were supported by the DFG's Center for Advancing Electronics Dresden (cfaed), EnhanceNano (No. 391979941), and the European Social Fund and the Federal State of Saxony (ESFProject "GRAPHD”), TU Dresden. T.D., A.N., and K.M. acknowledge the support by the Max Planck Society. G.B.B. acknowledges CNPq, Brazil, and Empa-Horizon 2020 Marie Skłodowska-Curie action COFUND for financial support. Finally, we acknowledge access to the Scanning Probe Microscopy User lab at Empa for the AFM measurements.

\section{REFERENCES}

(1) Cai, J.; Ruffieux, P.; Jaafar, R.; Bieri, M.; Braun, T.; Blankenburg, S.; Muoth, M.; Seitsonen, A. P.; Saleh, M.; Feng, X.; Müllen, K.; Fasel, R. Atomically Precise Bottom-up Fabrication of Graphene Nanoribbons. Nature 2010, 466, 470-473.

(2) Denk, R.; Hohage, M.; Zeppenfeld, P.; Cai, J.; Pignedoli, C. A.; Söde, H.; Fasel, R.; Feng, X.; Müllen, K.; Wang, S.; Prezzi, D.; Ferretti, A.; Ruini, A.; Molinari, E.; Ruffieux, P. Exciton-Dominated Optical Response of Ultra-Narrow Graphene Nanoribbons. Nat. Commun. 2014, 5, 4253.

(3) Llinas, J. P.; Fairbrother, A.; Barin, G. B.; Shi, W.; Lee, K.; Wu, S.; Choi, B. Y.; Braganza, R.; Lear, J.; Kau, N.; Choi, W.; Chen, C.; Pedramrazi, Z.; Dumslaff, T.; Narita, A.; Feng, X.; Müllen, K.; Fischer, F.; Zettl, A.; Ruffieux, P.; Yablonovitch, E.; Crommie, M.; Fasel, R.; Bokor, J. Short-Channel Field-Effect Transistors with 9-Atom and 13Atom Wide Graphene Nanoribbons. Nat. Commun. 2017, 8, 633.

(4) Fairbrother, A.; Sanchez-Valencia, J.-R.; Lauber, B.; Shorubalko, I.; Ruffieux, P.; Hintermann, T.; Fasel, R. High Vacuum Synthesis and Ambient Stability of Bottom-up Graphene Nanoribbons. Nanoscale 2017, 9, 2785-2792.

(5) Talirz, L.; Söde, H.; Dumslaff, T.; Wang, S.; Sanchez-Valencia, J. R.; Liu, J.; Shinde, P.; Pignedoli, C. A.; Liang, L.; Meunier, V.; Plumb, N. C.; Shi, M.; Feng, X.; Narita, A.; Müllen, K.; Fasel, R.; Ruffieux, P. On-Surface Synthesis and Characterization of 9-Atom Wide Armchair Graphene Nanoribbons. ACS Nano 2017, 11, 1380-1388.

(6) Ruffieux, P.; Wang, S.; Yang, B.; Sánchez-Sánchez, C.; Liu, J.; Dienel, T.; Talirz, L.; Shinde, P.; Pignedoli, C. A.; Passerone, D.;
Dumslaff, T.; Feng, X.; Müllen, K.; Fasel, R. On-Surface Synthesis of Graphene Nanoribbons with Zigzag Edge Topology. Nature 2016, $531,489-492$.

(7) Talirz, L.; Ruffieux, P.; Fasel, R. On-Surface Synthesis of Atomically Precise Graphene Nanoribbons. Adv. Mater. 2016, 28, $6222-6231$.

(8) Bennett, P. B.; Pedramrazi, Z.; Madani, A.; Chen, Y.-C.; de Oteyza, D. G.; Chen, C.; Fischer, F. R.; Crommie, M. F.; Bokor, J. Bottom-up Graphene Nanoribbon Field-Effect Transistors. Appl. Phys. Lett. 2013, 103, 253114.

(9) Li, X.; Zhu, Y.; Cai, W.; Borysiak, M.; Han, B.; Chen, D.; Piner, R. D.; Colombo, L.; Ruoff, R. S. Transfer of Large-Area Graphene Films for High-Performance Transparent Conductive Electrodes. Nano Lett. 2009, 9, 4359-4363.

(10) Wang, Y.; Zheng, Y.; Xu, X.; Dubuisson, E.; Bao, Q.; Lu, J.; Loh, K. P. Electrochemical Delamination of CVD-Grown Graphene Film: Toward the Recyclable Use of Copper Catalyst. ACS Nano 2011, 5, 9927-9933.

(11) Bae, S.; Kim, H.; Lee, Y.; Xu, X.; Park, J.-S.; Zheng, Y.; Balakrishnan, J.; Lei, T.; Ri, Kim H.; Song, Y. I.; Kim, Y. J.; Kim, K. S.; Özyilmaz, B.; Ahn, J. H.; Hong, B. H.; Iijima, S. Roll-to-Roll Production of 30-Inch Graphene Films for Transparent Electrodes. Nat. Nanotechnol. 2010, 5, 574-578.

(12) Lin, W.-H.; Chen, T.-H.; Chang, J.-K.; Taur, J.-I.; Lo, Y.-Y.; Lee, W.-L.; Chang, C.-S.; Su, W.-B.; Wu, C.-I. A Direct and PolymerFree Method for Transferring Graphene Grown by Chemical Vapor Deposition to Any Substrate. ACS Nano 2014, 8, 1784-1791.

(13) Kang, J.; Shin, D.; Bae, S.; Hong, B. H. Graphene Transfer: Key for Applications. Nanoscale 2012, 4, 5527.

(14) Hallam, T.; Berner, N. C.; Yim, C.; Duesberg, G. S. Strain, Bubbles, Dirt, and Folds: A Study of Graphene Polymer-Assisted Transfer. Adv. Mater. Interfaces 2014, 1, 1400115.

(15) Chen, Z.; Zhang, W.; Palma, C.-A.; Lodi, R. A.; Liu, B.; Abbas, A.; Richter, N.; Martini, L.; Wang, X.-Y.; Cavani, N.; Lu, H.; Mishra, N.; Coletti, C.; Berger, R.; Klappenberger, F.; Kläui, M.; Candini, A.; Affronte, M.; Zhou, C.; De Renzi, V.; del Pennino, U.; Barth, J. V.; Räder, H. J.; Narita, A.; Feng, X.; Müllen, K. Synthesis of Graphene Nanoribbons by Ambient-Pressure Chemical Vapor Deposition and Device Integration. J. Am. Chem. Soc. 2016, 138, 15488-15496.

(16) Cai, J.; Pignedoli, C. A.; Talirz, L.; Ruffieux, P.; Söde, H.; Liang, L.; Meunier, V.; Berger, R.; Li, R.; Feng, X.; Müllen, K.; Fasel, R. Graphene Nanoribbon Heterojunctions. Nat. Nanotechnol. 2014, 9, 896-900.

(17) Borin, B. G.; Song, Y.; de Fátima, G. I.; Souza, F. A. G.; Barreto, L. S.; Kong, J. Optimized Graphene Transfer: Influence of Polymethylmethacrylate (PMMA) Layer Concentration and Baking Time on Graphene Final Performance. Carbon 2015, 84, 82-90.

(18) Peltekis, N.; Kumar, S.; McEvoy, N.; Lee, K.; Weidlich, A.; Duesberg, G. S. The Effect of Downstream Plasma Treatments on Graphene Surfaces. Carbon 2012, 50, 395-403.

(19) Maghsoumi, A.; Brambilla, L.; Castiglioni, C.; Müllen, K.; Tommasini, M. Overtone and Combination Features of G and D Peaks in Resonance Raman Spectroscopy of the $\mathrm{C}_{78} \mathrm{H}_{26}$ Polycyclic Aromatic Hydrocarbon. J. Raman Spectrosc. 2015, 46, 757-764.

(20) Tommasini, M.; Castiglioni, C.; Zerbi, G. Raman Scattering of Molecular Graphenes. Phys. Chem. Chem. Phys. 2009, 11, 10185.

(21) Malard, L. M.; Pimenta, M. A.; Dresselhaus, G.; Dresselhaus, M. S. Raman Spectroscopy in Graphene. Phys. Rep. 2009, 473, 51-87.

(22) Saito, R.; Furukawa, M.; Dresselhaus, G.; Dresselhaus, M. S. Raman Spectra of Graphene Ribbons. J. Phys.: Condens. Matter 2010, 22, 334203.

(23) Eckmann, A.; Felten, A.; Verzhbitskiy, I.; Davey, R.; Casiraghi, C. Raman Study on Defective Graphene: Effect of the Excitation Energy, Type, and Amount of Defects. Phys. Rev. B: Condens. Matter Mater. Phys. 2013, 88, 035426.

(24) Thomsen, C.; Reich, S. Double Resonant Raman Scattering in Graphite. Phys. Rev. Lett. 2000, 85, 5214-5217.

(25) Verzhbitskiy, I. A.; Corato, M. D.; Ruini, A.; Molinari, E.; Narita, A.; Hu, Y.; Schwab, M. G.; Bruna, M.; Yoon, D.; Milana, S.; 
Feng, X.; Müllen, K.; Ferrari, A. C.; Casiraghi, C.; Prezzi, D. Raman Fingerprints of Atomically Precise Graphene Nanoribbons. Nano Lett. 2016, 16, 3442-3447.

(26) Castiglioni, C.; Tommasini, M.; Zerbi, G. Raman Spectroscopy of Polyconjugated Molecules and Materials: Confinement Effect in One and Two Dimensions. Philosophical Transactions of the Royal Society of London A: Mathematical, Physical and Engineering Sciences. 2004, 362, 2425-2459.

(27) Casiraghi, C.; Prezzi, D. Raman Spectroscopy of Graphene Nanoribbons: A Review. In GraphITA: Selected papers from the Workshop on Synthesis, Characterization and Technological Exploitation of Graphene and 2D Materials Beyond Graphene; Morandi, V., Ottaviano, L., Eds.; Springer International Publishing: Cham, 2017; pp 19-130.

(28) Gillen, R.; Mohr, M.; Thomsen, C.; Maultzsch, J. Vibrational Properties of Graphene Nanoribbons by First-Principles Calculations. Phys. Rev. B: Condens. Matter Mater. Phys. 2009, 80, 155418.

(29) Levshov, D. I.; Tran, H. N.; Paillet, M.; Arenal, R.; Than, X. T.; Zahab, A. A.; Yuzyuk, Y. I.; Sauvajol, J.-L.; Michel, T. Accurate Determination of the Chiral Indices of Individual Carbon Nanotubes by Combining Electron Diffraction and Resonant Raman Spectroscopy. Carbon 2017, 114, 141-159.

(30) Vandescuren, M.; Hermet, P.; Meunier, V.; Henrard, L.; Lambin, P. Theoretical Study of the Vibrational Edge Modes in Graphene Nanoribbons. Phys. Rev. B: Condens. Matter Mater. Phys. 2008, 78, 195401.

(31) Sanders, G. D.; Nugraha, A. R. T.; Saito, R.; Stanton, C. J. Coherent Radial-Breathing-like Phonons in Graphene Nanoribbons. Phys. Rev. B: Condens. Matter Mater. Phys. 2012, 85, 205401.

(32) Prezzi, D.; Varsano, D.; Ruini, A.; Marini, A.; Molinari, E. Optical Properties of Graphene Nanoribbons: The Role of ManyBody Effects. Phys. Rev. B: Condens. Matter Mater. Phys. 2008, 77, 041404.

(33) Osella, S.; Narita, A.; Schwab, M. G.; Hernandez, Y.; Feng, X.; Müllen, K.; Beljonne, D. Graphene Nanoribbons as Low Band Gap Donor Materials for Organic Photovoltaics: Quantum Chemical Aided Design. ACS Nano 2012, 6, 5539-5548.

(34) Piscanec, S.; Lazzeri, M.; Mauri, F.; Ferrari, A. C.; Robertson, J. Kohn Anomalies and Electron-Phonon Interactions in Graphite. Phys. Rev. Lett. 2004, 93, 185503.

(35) Liu, C.; Ma, Y.; Li, W.; Dai, L. The Evolution of Raman Spectrum of Graphene with the Thickness of $\mathrm{SiO}_{2}$ Capping Layer on Si Substrate. Appl. Phys. Lett. 2013, 103, 213103.

(36) Wang, Y. Y.; Ni, Z. H.; Shen, Z. X.; Wang, H. M.; Wu, Y. H. Interference Enhancement of Raman Signal of Graphene. Appl. Phys. Lett. 2008, 92, 043121.

(37) Senkovskiy, B. V.; Pfeiffer, M.; Alavi, S. K.; Bliesener, A.; Zhu, J.; Michel, S.; Fedorov, A. V.; German, R.; Hertel, D.; Haberer, D.; Petaccia, L.; Fischer, F. R.; Meerholz, K.; van Loosdrecht, P. H. M.; Lindfors, K.; Grüneis, A. Making Graphene Nanoribbons Photoluminescent. Nano Lett. 2017, 17, 4029-4037.

(38) Horcas, I.; Fernández, R.; Gómez-Rodríguez, J. M.; Colchero, J.; Gómez-Herrero, J.; Baro, A. M. WSXM: A Software for Scanning Probe Microscopy and a Tool for Nanotechnology. Rev. Sci. Instrum. 2007, 78, 013705.

(39) Kresse, G.; Furthmüller, J. Efficiency of Ab-Initio Total Energy Calculations for Metals and Semiconductors Using a Plane-Wave Basis Set. Comput. Mater. Sci. 1996, 6, 15-50.

(40) Parlinski, K. PHONON Software, 2010.

(41) Liang, L.; Meunier, V. First-Principles Raman Spectra of $\mathrm{MoS}_{2}$, $\mathrm{WS}_{2}$ and Their Heterostructures. Nanoscale 2014, 6, 5394-5401.

(42) del Corro, E.; Botello-Méndez, A.; Gillet, Y.; Elias, A. L.; Terrones, H.; Feng, S.; Fantini, C.; Rhodes, D.; Pradhan, N.; Balicas, L.; Gonze, X.; Charlier, J. C.; Terrones, M.; Pimenta, M. A. Atypical Exciton-Phonon Interactions in $\mathrm{WS}_{2}$ and $\mathrm{WSe}_{2}$ Monolayers Revealed by Resonance Raman Spectroscopy. Nano Lett. 2016, 16, 2363-2368. 